\title{
Layer-by-Layer Evolution of a Two-Dimensional Electron Gas Near an Oxide Interface
}

\author{
Young Jun Chang, ${ }^{1,2,3}$ Luca Moreschini, ${ }^{1}$ Aaron Bostwick, ${ }^{1}$ Geoffrey A. Gaines, ${ }^{1}$ Yong Su Kim, ${ }^{1}$ Andrew L. Walter, ${ }^{1,2}$ \\ Byron Freelon, ${ }^{1}$ Antonello Tebano, ${ }^{4}$ Karsten Horn, ${ }^{2}$ and Eli Rotenberg ${ }^{1, *}$ \\ ${ }^{1}$ Advanced Light Source (ALS), E. O. Lawrence Berkeley National Laboratory, Berkeley, California 94720, USA \\ ${ }^{2}$ Department of Physical Chemistry, Fritz-Haber-Institut der Max-Planck-Gesellschaft, Faradayweg 4-6, 14195 Berlin, Germany \\ ${ }^{3}$ Department of Physics, University of Seoul, Seoul 130-743, Korea \\ ${ }^{4}$ CNR-SPIN and Department of Civil Engineering and Computer Science Engineering, University of Roma Tor Vergata, \\ 00133 Rome, Italy \\ (Received 29 May 2013; published 18 September 2013)
}

\begin{abstract}
We report the momentum-resolved measurement of a two-dimensional electron gas at the $\mathrm{LaTiO}_{3} / \mathrm{SrTiO}_{3}$ interface by angle-resolved photoemission spectroscopy (ARPES). Thanks to an advanced sample preparation technique, the orbital character of the conduction electrons and the electronic correlations can be accessed quantitatively as each unit cell layer is added. We find that all of these quantities change dramatically with distance from the interface. These findings open the way to analogous studies on other heterostructures, which are traditionally a forbidden field for ARPES.
\end{abstract}

DOI: 10.1103/PhysRevLett.111.126401

PACS numbers: 71.20. $-\mathrm{b}, 73.20 .-\mathrm{r}, 79.60 . \mathrm{Jv}$

Interfaces between transition metal oxides provide an exciting field of research because of the numerous unusual physical phenomena they exhibit [1]. The behavior of correlated electrons in oxides is governed by the interplay of the carrier density $\rho$, the hopping integral $t$, the on-site Coulomb repulsion energy $U$, and the exchange energy $J$. At the interface, where these parameters differ with respect to the bulk, a space-charge layer may change the carrier density and induce new electronic phases. The singleparticle picture, successful in semiconductor physics, obviously fails to correctly describe the electronic structure at the surface or interface, as it often does in the bulk [2].

Recent progress in oxide film growth has made it possible to design heterostructures with atomically sharp interfaces. A remarkable example is the junction between the Mott insulator $\mathrm{LaTiO}_{3}$ and the band insulator $\mathrm{SrTiO}_{3}$, which shows 2D metallic conductivity [3], as verified by optical and angle-integrated photoemission spectroscopy [4,5], and superconductivity below $1 \mathrm{~K}$ [6]. Furthermore, the proposed theoretical descriptions suggest the presence of interface subbands involved in both magnetism and orbital ordering [7-9], which has crucial implications for high-temperature superconductivity and colossal magnetoresistance [10].

Whereas many experimental studies explored the detailed properties of this conductive channel, its investigation by high-resolution angle-resolved photoemission spectroscopy (ARPES) is still missing. In fact, although in principle able to fully unveil the electronic structure of materials, photoemission is surface sensitive and therefore cannot penetrate the top few layers to access a buried interface. An option frequently chosen in synchrotron beam lines is to work at the $\mathrm{Ti} L$ edge where the $\mathrm{Ti} 3 d$ signal is resonantly enhanced. However, this comes at the price of moderate [11] or no momentum resolution $[12,13]$. Two groups have recently tried to overcome this limitation by measuring the electronic states arising at the bare surface of cleaved $\mathrm{SrTiO}_{3}$ single crystals after induction of defects [14,15], but no high-resolution angle-resolved experiment has been done on the actual interface state. Here we choose a novel approach as we build epitaxially the heterostructure one unit cell at a time and we measure its band structure separately at each stage of the growth, thereby revealing for the first time the layer-by-layer evolution of the electronic states at the surface.

The samples were grown by pulsed laser deposition (PLD) and transferred in situ to the ARPES chamber of the beam line 7.0.1 end station at the Advanced Light Source [16]. Figure 1 shows a schematic picture of the heterostructure prepared. A Nb-doped $\mathrm{SrTiO}_{3}$ single crystal is used as a substrate in order to prevent charging during the ARPES experiment and covered with a thick $\mathrm{SrTiO}_{3}$ buffer layer to serve as a defect-free, well-ordered, intrinsically insulating substrate. Then the sample is prepared in a steplike fashion by means of a shutter translated during the PLD growth, with an $\mathrm{LaTiO}_{3}$ layer $(L 1)$ followed by layers of $\mathrm{SrTiO}_{3}(L 1 S n)$ to give an $\mathrm{SrTiO}_{3}(n) / \mathrm{LaTiO}_{3} / \mathrm{SrTiO}_{3}$ sandwich structure of different and controlled $\mathrm{SrTiO}_{3}$ thickness $(n=0-3)$ along the sample surface.

We focus in this report on the evolution in the electronic structure near the Fermi level $\left(E_{\mathrm{F}}\right)$ as a function of the distance from the surface to the underlying $\mathrm{LaTiO}_{3} /$ $\mathrm{SrTiO}_{3}$ interface. In the insets of Fig. 1, the constant-energy maps at $E_{\mathrm{F}}$ and the schematic Fermi surfaces change as we stack the $\mathrm{SrTiO}_{3}$ layers. Because of the short probing depth (a few angstroms) of photoemission typical for perovskite oxides at these low photon energies [17], the measured signal is strongly dominated by the first unit cell at the surface. This is also consistent with the rapid evolution measured on the core level intensities [16]. Therefore, we probe in case $L 1$ [Fig. 1(a)] the states of the $\mathrm{LaTiO}_{3}$ layer and in cases $L 1 S n$ 
(a)

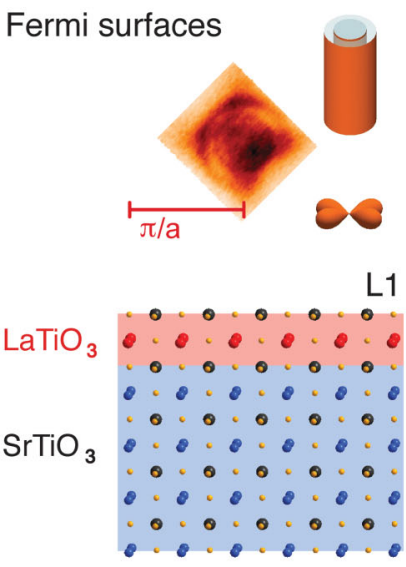

(b)

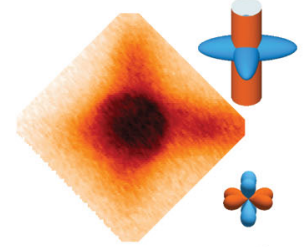

L1S1

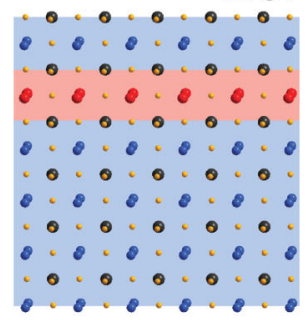

(c)

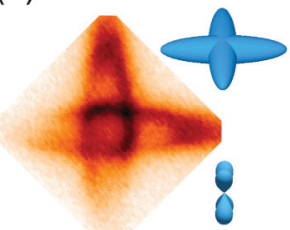

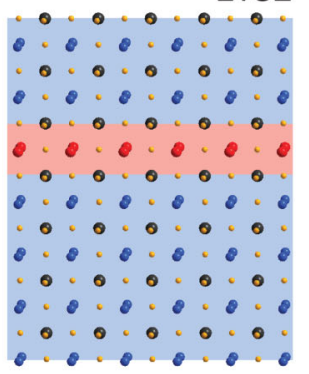

(d)

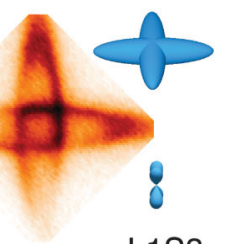

L1S3

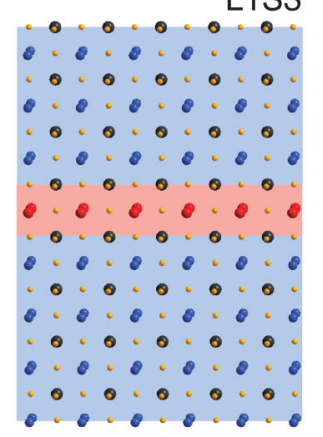

FIG. 1 (color). Experimental constant-energy maps at $E_{\mathrm{F}}$ and schematics of the Fermi surfaces at the vacuum interface of the $\mathrm{SrTiO}_{3}(n) / \mathrm{LaTiO}_{3} / \mathrm{SrTiO}_{3}(n=0-3$; sample $L 1$ or $L 1 S n)$ sandwich structures. Notice the clear change of the Fermi surface, corresponding to the transition from Ti $3 d_{x y}$ (orange) to $3 d_{x z, y z}$ (blue) oriented states ( $\mathrm{SrTiO}_{3}$ lattice constant $a=3.905 \AA$ and $\pi / a=$ $0.805 \AA^{-1}$ ). At the bottom, corresponding microscopic structures of the samples are illustrated by adding unit cell layers of $\mathrm{LaTiO}_{3}$ (red shade) and $\mathrm{SrTiO}_{3}$ (blue shade) on the $\mathrm{SrTiO}_{3}$ buffer layers [ $\mathrm{La}$ (red), $\mathrm{Sr}$ (blue), $\mathrm{Ti}$ (black), and oxygen (yellow)].

[Figs. 1(b)-1(d)] the states of the $n$th $\mathrm{SrTiO}_{3}$ layer from $\mathrm{LaTiO}_{3}$. This thickness-dependent electronic structure is not $a$ priori the same as the layer-dependent electronic structure of a thicker sample, with the difference being the additional perturbation of the structure due to the surface. However, a comparison between our data and the theory for the layer-dependent electronic structure of thicker samples [9] (calculations for each separate surface layer are not available) shows a fairly good agreement, which seems to indicate that the surface perturbation in the absence of defects is fairly minor. This is supported by the results of Ref. [18], where the bulk band structure of $\mathrm{SrTiO}_{3}$ without any apparent surface effects was measured.

A series of experimental constant-energy maps at the Fermi edge on samples $L 1$ to $L 1 S 3$ is shown in Figs. 2(a)-2(d). These measurements demonstrate the existence of a large

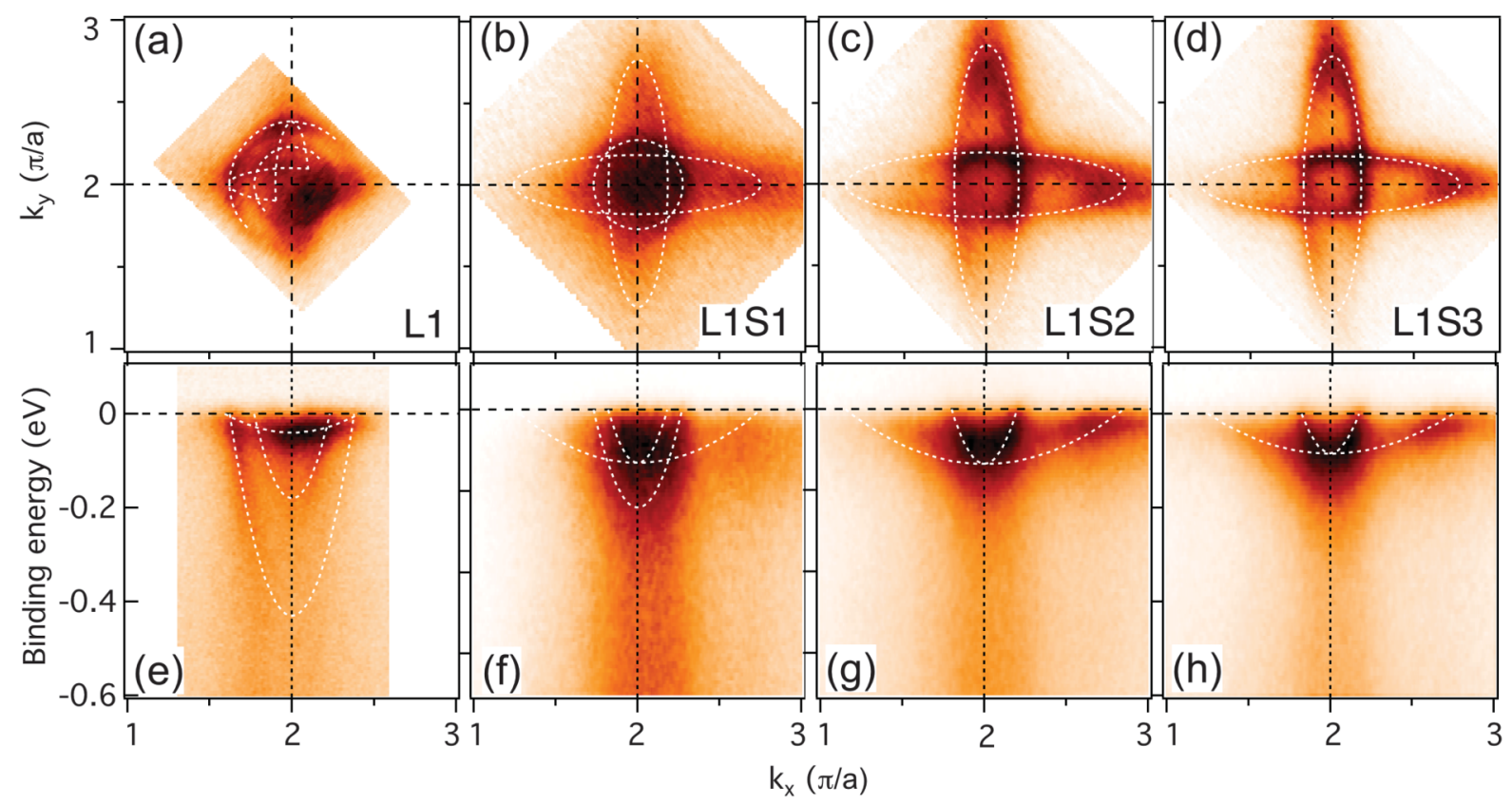

FIG. 2 (color). (a)-(d) Constant-energy maps of the states at $E_{\mathrm{F}}$ in the Brillouin zone at $\Gamma_{11}=(2 \pi$, $2 \pi)$ on samples $L 1$ to $L 1 S 3$. The circular (elliptic) contours are identified as the Fermi surfaces of the out-of-plane-oriented (in-plane-oriented) $d_{x y}\left(d_{x z, y z}\right)$ ellipsoidal pockets, respectively. (e)-(h) Energy dispersions along the horizontal dashed lines ( $\Gamma X$ direction) in (a)-(d) display the shift of the band bottom as well as the orbital occupancy switching. The white dotted lines are guides to the eye and mimic the band dispersions. (Measurement temperature $20 \mathrm{~K}$ with $h v=99 \mathrm{eV}$.) 
intrinsic population of charge carriers at the $\mathrm{LaTiO}_{3} / \mathrm{SrTiO}_{3}$ interface. The Fermi surface of sample $L 1$ [Fig. 2(a)] exhibits two concentric circular contours and two small ellipsoids. Consistently with other recent ARPES studies on bare $\mathrm{SrTiO}_{3}$ $[14,15,18,19]$, hereafter we assign the circular (elliptic) Fermi surfaces to $d_{x y}\left(d_{x z, y z}\right)$ electrons. These states are derived from atomic orbitals of the Ti atoms (black dots in Fig. 1). In our notation, $z$ is along the growth direction of the layers and $x, y$ lie in the interface plane. Before going further, we emphasize that the $\mathrm{SrTiO}_{3}$ buffer layer itself does not present any electronic state inside its $\sim 3.2 \mathrm{eV}$ band gap [16]. As a consequence, surface electrons induced by either vacuum cleavage [14] or x-ray exposure [15] in previous studies do not contribute to the intensity visible in the maps of Fig. 2, which originates purely from the interface states. Within our experimental error the two $d_{x y}$ features of Fig. 2(a) do not disperse along the $k_{z}$ direction when changing the wave vector in the 3D Brillouin zone from $\Gamma$ to $X$ [16]. This clearly demonstrates the 2D character of the two $d_{x y}$ bands.

The Fermi surface maps and band dispersions shown in Fig. 2 closely resemble the ones proposed in recent theoretical models of interface subbands [7-9]. For sample $L 1$, the measured band dispersion consists of a stronger intensity close to $E_{\mathrm{F}}$, due to the $d_{x z, y z}$ states, and of two weaker and more dispersive features, corresponding to $d_{x y}$ electrons. Their dispersion can be mimicked by two parabolas with minimum at a binding energy of $\sim 0.43$ and $\sim 0.18 \mathrm{eV}$ [white dotted lines in Fig. 2(e)] by analyzing data taken at different photon energies [16]. These bands extend to significantly higher binding energies than those reported for bare $\mathrm{SrTiO}_{3}(0.21$ and $0.11 \mathrm{eV})$ [14,15]. Instead, the lower band well matches the calculations for the same $\mathrm{LaTiO}_{3}$ layer embedded in $\mathrm{SrTiO}_{3}$ studied here (minimum at $\sim 0.4 \mathrm{eV}$ ), whereas the upper band can be assigned to the contribution of the underlying layer, of which the Ti $3 d_{x y}$ band minimum is expected at $0.2 \mathrm{eV}$ [9]. In addition, the ratio of the momentum-space areas of the $d_{x y}$ to $d_{x z, y z}$ bands, which by Luttinger's theorem corresponds to the relative orbital occupation, is much larger here than for the $\mathrm{SrTiO}_{3}$ surface states. Clearly, the depopulation of the $d_{x z, y z}$ states in favor of the the $d_{x y}$ band ("orbital order") is much stronger for the $\mathrm{LaTiO}_{3} / \mathrm{SrTiO}_{3}$ interface in comparison to the $\mathrm{SrTiO}_{3}$ surface states [14].

From the parabolic fit, we estimate the effective mass $m^{*} \simeq 0.8 m_{0}$ (lower band) and $m^{*} \simeq 0.7 m_{0}$ (upper band), considerably smaller than for the $d_{x y}$ band in bulk $\mathrm{SrTiO}_{3}$, i.e., $1.2 m_{0}$ [18]. Since the electron mobility is inversely proportional to the effective mass, the in-plane electron mobility is thus significantly enhanced at the interface layer, supporting the 2D high-mobility electron transport and superconductivity witnessed in this $[6,20]$ and similar interfaces [21,22].

The band structure shows a clear evolution with the successive deposition of $\mathrm{SrTiO}_{3}$ layers. Sample L1S1 shows a single circular contour and two larger crossing elliptic $d_{x z, y z}$ bands [Fig. 2(b)]. The $d_{x y}$ band has a similar size to that of the upper band in Fig. 2(a), which was assigned to the underlying $\mathrm{SrTiO}_{3}$ layer. This symmetry with respect to the $\mathrm{LaTiO}_{3}$ unit cell indicates that the role of surface to the electronic structure is relatively minor. In addition, we speculate that there is some signal (such as the central intensity of the small elliptic bands) from underneath $\mathrm{LaTiO}_{3}$. Note that in Fig. 2(b) the bands are considerably broader due to electron correlation, a point we treat more in detail later.

As $\mathrm{SrTiO}_{3}$ layers are further added, the $d_{x z, y z}$ bands have their major axis elongated nearly to the Brillouin zone boundary in $L 1 S 2$ and then slightly shrink in $L 1 S 3$, while the $d_{x y}$ band shifts entirely above the Fermi level and its circular Fermi surface disappears from the ARPES maps. Therefore, the occupation of $d_{x y}$ orbitals decreases and vanishes in favor of the $d_{x z, y z}$ orbitals as the distance from the interface increases. The direct spectroscopic measurement of the band structure at the surface of each structure allows one to determine the unit-cell-dependent occupancies of the different $d$ orbitals, as shown in Fig. 3(a). This quantity is the ratio of the Fermi surface area to the area of the Brillouin zone, assuming degenerate spin states. For sample $L 1$, we count only the Fermi surface area of the bigger parabola in order to account for the $d_{x y}$ occupancy.

Our observations indicate that the electrons rearrange near the interface, with $d_{x y}$ states being more confined and $d_{x z, y z}$ states less confined near the interface. The evolution of the orbital occupation along the $z$ axis is schematically represented by the different sizes of the orbitals, i.e., $d_{x y}$
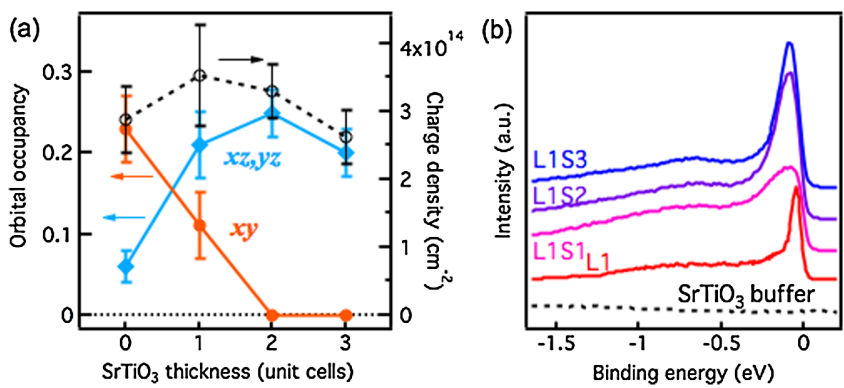

FIG. 3 (color online). (a) Surface layer occupancies of the Ti $3 d$ orbitals ( $d_{x y}$, filled circles; $d_{x z, y z}$, filled diamonds) and charge density (per $\mathrm{cm}^{2}$ at the surface layer; open squares) of individual unit-cell layers near the interface. At the charge-donating $\mathrm{LaTiO}_{3}$ layer $\left(\mathrm{SrTiO}_{3}\right.$ thickness $\left.=0\right)$ the $d_{x y}$ are the dominant states, while for increasing $\mathrm{SrTiO}_{3}$ thickness the electrons acquire more and more $d_{x z, y z}$ character. At $L 1 S 2$ there is no longer a trace of the $d_{x y}$ states at $E_{\mathrm{F}}$. By taking into account the degeneracy between $d_{x z}$ and $d_{y z}$ orbitals, the total occupancy of the $d_{x z, y z}$ states is twice the value shown here. The overall surface charge density shows little variation $(<20 \%)$, comparable with the error bar of the measurement. Note that the error bars are largest for sample $L 1 S 1$, where the contours have the worst definition. (b) Energy distribution curves in correspondence of $\Gamma_{11}$, showing the reduction in the quasiparticle weight for sample $L 1 S 1$ and the absence of any intensity at $E_{\mathrm{F}}$ in the $\mathrm{SrTiO}_{3}$ buffer layer. 
(orange) and $d_{x z, y z}$ (blue) in the sketches of Fig. 1. Quantitative discrepancies are present with respect to the theoretical predictions [9]: In the first layer, the $d_{x y}$ population (for both $2 \mathrm{D}$ bands) is larger than expected $(\sim 0.23$ against $\sim 0.18$ ) but very similar for the $d_{x z, y z}$ states $(\sim 0.06)$. In addition, the maximum occupation for the $d_{x z, y z}$ states is shifted by one unit cell, i.e., from the first to the second $\mathrm{SrTiO}_{3}$ layer. Note that, while the charges are transferred from $d_{x y}$ to $d_{x z, y z}$ states, the total electron density at the surface plane remains fairly constant, within the measurement error bars, in the thickness range under investigation.

The $d_{x y}$ orbital ordering directly affects the electron mobility. Indeed, the presence of a single $m^{*}$ for the inplane conductivity of the $d_{x y}$ states, as opposed to two different effective masses along the long and short axes of the ellipsoid for the $d_{x z, y z}$ bands, supports a multiple charge carrier model suggested by transport measurements $[20,23]$. It is worthwhile to note that the presence of a small effective mass of $d_{x z, y z}$ electrons along $k_{y}$ and $k_{x}$, respectively, can account for the thicker conductive layer observed there ( 3-10 unit cells) [20], compared to the effective spread of the $d_{x y}$ states over $\sim 1-2$ unit cells as shown here. This may be a useful term of comparison for the $\mathrm{LaAlO}_{3} / \mathrm{SrTiO}_{3}$ interface, where transport gives an extension for the 2D electron gas of a few nanometers $[24,25]$ as opposed to 1 unit cell as inferred by photoelectron spectroscopy [26].

The charge densities extracted in this work are about one order of magnitude larger than recently found by transport experiments [27]. However, this is in line with the lower oxygen pressure used in this work for the film growth [16], which is known to strongly affect the carrier concentration [28]. Equally large discrepancies are present within the transport data available for the similar $\mathrm{LaAlO}_{3} / \mathrm{SrTiO}_{3}$ interface due to controllable and noncontrollable differences in the growth conditions and possibly in the sample storage environment between the growth and the experiment [25,28-30]. Performing measurements on samples of different thickness but grown in parallel and on the same substrate adds consistency to the comparison and rules out extrinsic origins as a source of the observed relative variations. We note that the total charge density at $L 1$ of 0.44 electrons per $\mathrm{Ti}$ atom $(e / \mathrm{Ti})$ found here is similar to previous electron energy loss spectroscopy data (EELS), yielding $0.3 e / \mathrm{Ti}$ in Ref. [27] and $0.24 e / \mathrm{Ti}$ in Ref. [27]. However, EELS is sensitive both to itinerant and localized charges which we do not account for in our derivation, so the similarity is likely to be purely fortuitous.

Another clear difference with respect to transport data is the absence of a critical thickness for the metallic channel in our data, in contrast with previous experiments indicating insulating behavior for thin $\mathrm{SrTiO}_{3}$ layers on $\mathrm{LaTiO}_{3} / \mathrm{SrTiO}_{3}$ [27]. As hypothesized in that study, the absence of a metallic state for low coverages is likely to be due to extrinsic reasons, namely, the presence of surface adsorbates acting as localization centers for the electrons, which we avoid in this in situ experiment. Such a hypothesis is supported by an independent work showing that surface absorbates can indeed modulate the metallic state in $\mathrm{LaAlO}_{3} / \mathrm{SrTiO}_{3}$ [30].

In addition to a layer-dependent orbital ordering, dimensionality, and charge distribution, our results also suggest an evolution in the degree of electron correlation, witnessed by the presence of a tail of the states from the bottom of the bands towards higher binding energy. Such a tail reflects the presence of multiparticle excitations, the so-called incoherent part of the ARPES spectral function [31]. This tail is present in all the samples but is most prominent in sample $L 1 S 1$ [Fig. 2(f)]. The presence of the tail is inversely correlated to the sharpness of the bands near $E_{\mathrm{F}}$, with sample $L 1 S 1$ having the least sharp (and hence weakest) quasiparticle features. Since the samples are grown on the same substrate under the same conditions (and in fact the $L 1 S 1$ layer is present under the subsequent $\mathrm{SrTiO}_{3}$ layers), it is difficult to explain this behavior in terms of variations of the sample quality. Rather, they are likely to reflect an intrinsic evolution in the correlation strength with overlayer thickness.

Although we cannot derive the actual details of the buried interface states in $L 1 S n$ samples of large $n$, our results suggest the possibility that, although the induced charge distribution extends over several layers to either side of $\mathrm{LaTiO}_{3}$, the coherent quasiparticles are confined much closer to the $\mathrm{LaTiO}_{3}$ interface. In the first unit cell deposited upon the $\mathrm{LaTiO}_{3}$ interface, the coherence length abruptly drops. The $L 1 S 1$ sample in fact appears to represent the highest-correlation boundary between the $L 1$ layer carrying the coherent quasiparticles of the interface and the $L 1 S n(n>1)$ layers, where for increasing $n$ the bands gradually evolve to the weakly correlated electrons of bulk $\mathrm{SrTiO}_{3}$. Note in this respect that the Fermi surface shrinks between $L 1 S 2$ and $L 1 S 3$, starting an estimated trend for increasing $n$ which would lead to a gradual vanishing of the Fermi surface and no metallic state, as expected in the undoped oxide.

Our work demonstrates the potential of a layer-by-layer investigation of oxide heterostructures by ARPES in order to determine the fundamental electronic properties in the interfaces. Covering the charge-donating $\mathrm{LaTiO}_{3}$ layer with an atomically controlled thickness of $\mathrm{SrTiO}_{3}$ overlayers, we directly observe the interface $d_{x y}$ orbital ordering as well as the 2D electronic subband structure (i.e., 2D electron liquid), in contrast to the noninteracting 2D electron gas at semiconductor interfaces [32]. The detailed investigation of the character of these electronic states is crucial for understanding the ground state phase diagram, which includes superconductivity $[6,22]$ and magnetism [23]. We believe that the present study can be extended in principle to all the other correlated oxide heterostructures of interest for the scientific community in recent years $[21,22,27,33-35]$ and can be effectively complemented in 
the future by spin-resolved photoemission for the study of spin-ordered phases.

The ALS is supported by the director of the Office of Science, Office of Basic Energy Sciences, of the U.S. Department of Energy under Contract No. DE-AC0205CH11231. Y.J.C., A. L. W., and K.H. acknowledge the support by the Max Planck Society. Y. J.C. acknowledges support from National Research Foundation of Korea under Grant No. NRF-2012R1A1A2043619. L. M. acknowledge support by a grant from the Swiss National Science Foundation (SNSF) (Project No. PBELP2-125484).

*erotenberg@lbl.gov

[1] J. Mannhart and D. G. Schlom, Science 327, 1607 (2010).

[2] G. Kotliar and D. Vollhardt, Phys. Today 57, No. 3, 53 (2004).

[3] A. Ohtomo, D. A. Muller, J. L. Grazul, and H. Y. Hwang, Nature (London) 419, 378 (2002).

[4] S. S. A. Seo, W. S. Choi, H. N. Lee, L. Yu, K. W. Kim, C. Bernhard, and T.W. Noh, Phys. Rev. Lett. 99, 266801 (2007).

[5] M. Takizawa, H. Wadati, K. Tanaka, M. Hashimoto, T. Yoshida, A. Fujimori, A. Chikamatsu, H. Kumigashira, M. Oshima, K. Shibuya, T. Mihara, T. Ohnishi, M. Lippmaa, M. Kawasaki, H. Koinuma, S. Okamoto, and A. J. Millis, Phys. Rev. Lett. 97, 057601 (2006).

[6] J. Biscaras, N. Bergeal, A. Kushwaha, T. Wolf, A. Rastogi, R. C. Budhani, and J. Lesueuer, Nat. Commun. 1, 89 (2010).

[7] S. Okamoto and A. J. Millis, Nature (London) 428, 630 (2004).

[8] R. Pentcheva and W. E. Pickett, Phys. Rev. Lett. 99, 016802 (2007).

[9] P. Larson, Z. S. Popovic, and S. Satpathy, Phys. Rev. B 77, 245122 (2008).

[10] Y. Tokura and N. Nagaosa, Science 288, 462 (2000).

[11] G. Berner, M. Sing, H. Fujiwara, A. Yasui, Y. Saitoh, A. Yamasaki, Y. Nishitani, A. Sekiyama, N. Pavlenko, T. Kopp, C. Richter, J. Mannhart, S. Suga, and R. Claessen, Phys. Rev. Lett. 110, 247601 (2013).

[12] A. Koitzsch, J. Ocker, M. Knupfer, M. C. Dekker, K. Dörr, B. Büchner, and P. Hoffmann, Phys. Rev. B 84, 245121 (2011).

[13] C. Cancellieri, M. L. Reinle-Schmitt, M. Kobayashi, V. N. Strocov, T. Schmitt, P. R. Willmott, S. Gariglio, and J.-M. Triscone, Phys. Rev. Lett. 110, 137601 (2013).

[14] A. F. Santander-Syro, O. Copie, T. Kondo, F. Fortuna, S. Pailhes, R. Weht, X. G. Qiu, F. Bertran, A. Nicolaou, A. Taleb-Ibrahimi,P. Le Fevre, G. Herranz, M. Bibes, N. Reyren, Y. Apertet, P. Lecoeur, A. Barthelemy, and M. J. Rozenberg, Nature (London) 469, 189 (2011).

[15] W. Meevasana, P.D.C. King, R. H. He, S.-K. Mo, M. Hashimoto, A. Tamai, P. Songsiriritthigul, F. Baumberger, and Z.-X. Shen, Nat. Mater. 10, 114 (2011).

[16] See Supplemental Material at http://link.aps.org/ supplemental/10.1103/PhysRevLett.111.126401 for details on the film growth and spectroscopic measurements.
[17] M. A. Hossain, J. D.F. Mottershead, D. Fournier, A. Bostwick, J.L. McChesney, E. Rotenberg, R. Liang, W. N. Hardy, G. A. Sawatzky, I. S. Elfimov, D. A. Bonn, and A. Damascelli, Nat. Phys. 4, 527 (2008).

[18] Y.J. Chang, A. Bostwick, Y.S. Kim, K. Horn, and E. Rotenberg, Phys. Rev. B 81, 235109 (2010).

[19] W. Meevasana, X. J. Zhou, B. Moritz, C.-C. Chen, R. H. He, S.-I. Fujimori, D. H. Lu, S.-K. Mo, R. G. Moore, F. Baumberger, T.P. Devereaux, D. van der Marel, N. Nagaosa, J. Zaanen, and Z.-X. Shen, New J. Phys. 12, 023004 (2010).

[20] J. S. Kim, S. S. A. Seo, M.F. Chisholm, R. K. Kremer, H.-U. Habermeier, B. Keimer, and H. N. Lee, Phys. Rev. B 82, 201407 (2010).

[21] A. Ohtomo and H. Y. Hwang, Nature (London) 427, 423 (2004).

[22] N. Reyren, S. Thiel, A. D. Caviglia, L.F. Kourkoutis, G. Hammerl, C. Richter, C. W. Schneider, T. Kopp, A. S. Ruetschi, D. Jaccard, M. Gabay, D. A. Muller, J. M. Triscone, and J. Mannhart, Science 317, 1196 (2007).

[23] Ariando, X. Wang, G. Baskaran, Z. Q. Liu, J. Huijben, J. B. Yi, A. Annadi, A. R. Barman, A. Rusydi, S. Dhar, Y.P. Feng, J. Ding, H. Hilgenkamp, and T. Venkatesan, Nat. Commun. 2, 188 (2011).

[24] M. Basletic, J. L. Maurice, C. Carretero, G. Herranz, O. Copie, M. Bibes, E. Jacquet, K. Bouzehouane, S. Fusil, and A. Barthelemy, Nat. Mater. 7, 621 (2008).

[25] W. Siemons, G. Koster, H. Yamamoto, W. A. Harrison, G. Lucovsky, T.H. Geballe, D.H. A. Blank, and M.R. Beasley, Phys. Rev. Lett. 98, 196802 (2007).

[26] M. Sing, G. Berner, K. Goß, A. Müller, A. Ruff, A. Wetscherek, S. Thiel, J. Mannhart, S. A. Pauli, C.W. Schneider, P. R. Willmott, M. Gorgoi, F. Schäfers, and R. Claessen, Phys. Rev. Lett. 102, 176805 (2009).

[27] H. W. Jang, D. A. Felker, C. W. Bark, Y. Wang, M. K. Niranjan, C. T. Nelson, Y. Zhang, D. Su, C. M. Folkman, S. H. Baek, S. Lee, K. Janicka, Y. Zhu, X. Q. Pan, D. D. Fong, E. Y. Tsymbal, M.S. Rzchowski, and C. B. Eom, Science 331, 886 (2011).

[28] A. Kalabukhov, R. Gunnarsson, J. Börjesson, E. Olsson, T. Claeson, and D. Winkler, Phys. Rev. B 75, 121404 (2007).

[29] M. Huijben, A. Brinkman, G. Koster, G. Rijnders, H. Hilgenkamp, and D.H. A. Blank, Adv. Mater. 21, 1665 (2009).

[30] Y. Xie, Y. Hikita, C. Bell, and H. Y. Hwang, Nat. Commun. 2, 494 (2011).

[31] A. Damascelli, Z. Hussain, and Z.-X. Shen, Rev. Mod. Phys. 75, 473 (2003).

[32] S. N. Takeda, N. Higashi, and H. Daimon, Phys. Rev. Lett. 94, 037401 (2005).

[33] J. Chakhalian, J. W. Freeland, H.-U. Habermeier, G. Cristiani, G. Khaliullin, M. van Veenendaal, and B. Keimer, Science 318, 1114 (2007).

[34] G. Logvenov, A. Gozar, and I. Bozovic, Science 326, 699 (2009).

[35] E. Benckiser, M. W. Haverkort, S. Brck, E. Goering, S. Macke, A. Fra, X. Yang, O. K. Andersen, G. Cristiani, H.-U. Habermeier, A. V. Boris, I. Zegkinoglou, P. Wochner, H.-J. Kim, V. Hinkov, and B. Keimer, Nat. Mater. 10, 189 (2011). 